\title{
Spontaneous generation and reversal of helicity in anisotropic turbulence
}

\author{
Wesley Agoua* \\ CNRS, Univ Lyon, Ecole Centrale de Lyon, Univ Lyon 1 Claude Bernard, INSA Lyon, \\ Laboratoire de Mécanique des Fluides et d'Acoustique, UMR5509, 69134 Ecully, France \\ Benjamin Favier ${ }^{\circ}$ \\ Aix Marseille Univ, CNRS, Centrale Marseille, IRPHE UMR 7342, Marseille, France \\ Alexandre Delache \\ CNRS, Univ Lyon, Ecole Centrale de Lyon, Univ Lyon 1 Claude Bernard, INSA Lyon, \\ Laboratoire de Mécanique des Fluides et d'Acoustique, UMR5509, 69134 Ecully, France \\ and Université Jean Monnet de Saint Etienne, 42000 Saint Etienne, France \\ Antoine Briard 10 \\ CEA, DAM, DIF, F-91279 Arpajon, France \\ Wouter J. T. Bos (1) ${ }^{\dagger}$ \\ CNRS, Univ Lyon, Ecole Centrale de Lyon, Univ Lyon 1 Claude Bernard, INSA Lyon, \\ Laboratoire de Mécanique des Fluides et d'Acoustique, UMR5509, 69134 Ecully, France
}

(Received 24 November 2020; accepted 5 May 2021; published 8 June 2021)

\begin{abstract}
Helicity plays an important role in spectacular geophysical phenomena such as hurricanes or the generation of the terrestrial magnetic field. The present investigation shows how helicity can be created in a statistically homogeneous but anisotropic flow, driven by buoyancy. If the flow is close enough to a two-dimensional limit, spontaneous symmetry breaking leads to the generation of mean helicity. In particular, we explain these observations by identifying a simple linear mechanism, the relevance of which is illustrated by simulations of unstably stratified turbulence in a conducting fluid on which a magnetic field is imposed. Finally it is shown that the self-organized state displays dynamical reversals of the sign of the mean helicity.
\end{abstract}

DOI: 10.1103/PhysRevE.103.L061101

a. On the origin and importance of helicity. Helicity is associated with the corkscrew motion of fluid particles. The mean helicity [1] is a topological invariant of the Euler equations and is defined as

$$
H=\langle\boldsymbol{u} \cdot \boldsymbol{\omega}\rangle,
$$

where $\boldsymbol{u}$ is the velocity, $\boldsymbol{\omega}=\boldsymbol{\nabla} \times \boldsymbol{u}$ the vorticity, and the brackets denote an ensemble average. Its value measures the knottedness of the vortex lines in a fluid [2] and is zero in a mirror-symmetric flow.

The presence of helicity is presumably important for the generation of the Earth's magnetic field [3-5]. Furthermore, helicity is strong in hurricanes [6-8] and helical modes can be considered the building-blocks of the turbulent energy cascade of turbulent flows [9-13]. It has been known that the presence of helicity in isotropic turbulence does somewhat weaken the energy cascade [14-16]. These effects become more drastic and alter completely the energy cascade when strong helical forcing is used [17], a possibility anticipated some decades ago [18]. Also, in the presence of body forces

\footnotetext{
*wesley.agoua@ec-lyon.fr.

†wouter.bos@ec-lyon.fr
}

such as the buoyancy force or rotation, the influence of helicity can affect flow properties in a significant way $[19,20]$. Yet another interesting feature is that an inhomogeneous distribution of mean helicity can induce a mean flow [21,22].

Helicity can thus play an important role in turbulent flows. Both in laboratory experiments and numerical simulations aiming at the investigation of its effects, it is usual to inject helicity into a flow either by boundary conditions or by adding a helical volume force to the system. In the dynamo experiments of Riga and Karlsruhe [23,24], for example, a helical mean flow was imposed through the shape of the container. In the von Kármán sodium experiment [25], mean helicity is injected by counter-rotating impellers. In numerical simulations, helicity is often injected by adding an ABC flow containing mean helicity [26,27], or by starting from helical initial conditions $[28,29]$. Using artificial forcing combined with strong rotation, helicity can be generated in a more spontaneous manner [30]. Apart from this last reference, in most of the above cited investigations, helicity is thus artificially imposed and its influence on the system is subsequently assessed. In natural flows, a known source of helicity is Ekman pumping $[31,32]$, or the interplay of inertial wave packets with buoyancy $[33,34]$. 
However, in the absence of statistical inhomogeneity or artificial forcing, is it possible to generate helicity? In the present investigation, we will show how helicity can be generated spontaneously by symmetry breaking of an initially nonhelical system. It seems that the mechanism presented in this Letter is thereby fairly generic and a good candidate to explain the genesis of helical motion in a number of geo- and astrophysical flows.

In the following we will derive a simple model, involving the linear interaction of skew diffusion [a quantity defined below in Eq. (5)] and helicity. Analyzing this model, we will show that two ingredients are important for the current mechanism to operate: the presence of an unstable density stratification, and strong anisotropy.

b. Unstably stratified turbulence, skew diffusion, and helicity. The governing equations of unstably stratified turbulence in the presence of a uniform density gradient in the $z$ direction are, in the Boussinesq approximation,

$$
\begin{gathered}
\partial_{t} \boldsymbol{u}+\boldsymbol{u} \cdot \nabla \boldsymbol{u}=-\nabla p+v \Delta \boldsymbol{u}+\theta \boldsymbol{e}_{z}+\boldsymbol{F} / \rho_{0}, \\
\partial_{t} \theta+\boldsymbol{u} \cdot \nabla \theta=\kappa \Delta \theta-N^{2} \boldsymbol{u} \cdot \boldsymbol{e}_{z}, \\
\nabla \cdot \boldsymbol{u}=0,
\end{gathered}
$$

where $p$ is pressure divided by the mean density $\rho_{0}, v$ the kinematic viscosity, $\theta=\rho g / \rho_{0}$ the buoyancy perturbation, with $\rho$ the density fluctuation, $g$ the acceleration due to gravity, $\kappa$ the diffusivity, $N$ the Brunt-Väisälä frequency, and $\boldsymbol{F}$ an additional body force or damping term. Later on in this investigation we will apply a magnetic field to the system, in which case the term $\boldsymbol{F}$ is associated with the Lorentz force.

In statistically homogeneous, mirror-symmetric flow, in the presence of a constant mean density gradient, the large-scale dynamics of the system are characterized by the kinetic energy $\langle\boldsymbol{u} \cdot \boldsymbol{u}\rangle / 2$, buoyancy variance $\left\langle\theta^{2}\right\rangle$, and buoyancy flux $\left\langle u_{z} \theta\right\rangle$. These three quantities measure the evolution of the flow and the interaction of the density and velocity fields. When mirror symmetry is broken, which corresponds to the presence of mean helicity $H$, a new statistical correlation appears (zero in mirror-symmetric flow), the skew diffusion

$$
Q \equiv Q_{z}=\left\langle\theta \omega_{z}\right\rangle
$$

a quantity introduced by Moffatt [35], and somewhat forgotten since. However, in recent work this quantity was evaluated in isotropic helical turbulence on which a uniform passive scalar gradient is imposed [36].

An important insight, which motivates the present investigation, is that skew diffusion can be generated by a linear mechanism in the presence of helicity. From the evolution equations for $\boldsymbol{u}$ and $\theta$ [Eqs. (2)-(4)] we derive (in the absence of $\boldsymbol{F}$ ),

$$
\frac{d Q}{d t}=N^{2} H_{z}-D_{Q}-\epsilon_{Q},
$$

where the nonlinear damping and viscous terms are respectively defined by

$$
D_{Q}=-\left\langle\theta \boldsymbol{\omega} \cdot \nabla u_{z}\right\rangle, \quad \epsilon_{Q}=(\nu+\kappa)\left\langle\nabla \theta \cdot \nabla \omega_{z}\right\rangle .
$$

We thus see that the production term [first term in the righhand side of Eq. (6)] is directly proportional to the vertical component of the helicity $H_{z}=\left\langle u_{z} \omega_{z}\right\rangle$.

Analogously, when writing the equation for the helicity, it is observed that the skew diffusion appears as a production term,

$$
\frac{d H_{z}}{d t}=Q-D_{H}-\epsilon_{H}
$$

with

$$
D_{H}=\left\langle\omega_{z} \boldsymbol{e}_{z} \cdot \nabla p\right\rangle, \quad \epsilon_{H}=v\langle\nabla \omega: \nabla \boldsymbol{u}\rangle .
$$

The production term is not present if the scalar is passive. However, when back reaction through buoyancy is present, the system is thus piloted by a linear production mechanism [first terms on the right-hand side of Eqs. (6) and (8)]. The other terms in the system are nonlinear damping $D_{Q}, D_{H}$ and viscodiffusive terms $\epsilon_{Q}, \epsilon_{H}$.

In the absence of the nonlinear damping, we can expect growth of $H_{z}$ and $Q$ if diffusive effects are small enough. The whole question is thus whether the nonlinear damping terms are strong enough to annihilate the combined linear production of helicity and skew diffusion. There is an asymptotic limit where this should happen: from Eqs. (6)-(8) it can be deduced that a system invariant along $\boldsymbol{e}_{z}$ (hereafter called 2D3C for two-dimensional three components) will not be damped by the nonlinear terms.

Indeed, in the $2 \mathrm{D} 3 \mathrm{C}$ limit, the gradient of the velocity and pressure field reduces to $\nabla=\left(\partial_{x}, \partial_{y}, 0\right)^{T}$ and consequently $\boldsymbol{\omega}=\omega_{z} \boldsymbol{e}_{z}$. Therefore both $D_{H}$ and $D_{Q}$ are zero since $\boldsymbol{e}_{z} \cdot \boldsymbol{\nabla}$ vanishes. It seems therefore plausible that helicity (and skew diffusion) will be generated in systems close to the 2D3C limit [37]. While the buoyancy force is anisotropic, it is known to sustain fully three-dimensional turbulent states $[28,38,39]$ and is therefore probably not sufficient on its own to reach the 2D3C limit. In the present investigation we will consider both the case $\boldsymbol{F}=0$ and the case where this term is associated with an externally imposed magnetic field, allowing the flow to approach the 2D3C regime.

c. Results. Equations (2)-(4) are integrated using pseudospectral Direct Numerical Simulations. Details on the 12 simulations documented in this investigation are given in the Supplemental Material [40], which includes Ref. [47]. The typical Reynolds number based on the rms velocity fluctuation and integral length scale is varying in the range $R_{L} \in$ $\left[10^{3}, 10^{4}\right]$ for all runs with nonzero magnetic field. The initial value of the normalized helicity is small in all runs. All results are presented during a statistically steady state.

We first consider a reference case of buoyancy-driven turbulence without imposed magnetic field $[\boldsymbol{F}=\mathbf{0}$ in Eq. (2)]. Isoenstrophy surfaces are shown in Fig. 1(a) for the case $N=0.6$ illustrating the presence of small-scale flow structures. Even though the mechanism which generates the flow is inherently anisotropic, no generation of mean helicity was observed, irrespective of the value of $N$, which was varied in the range $N \in[0.3,4.5]$. These initial tests show that in statistically homogeneous buoyancy-driven turbulence without the presence of other effects, the strength of the nonlinear damping is too large to allow for a spontaneous generation of mean helicity. 


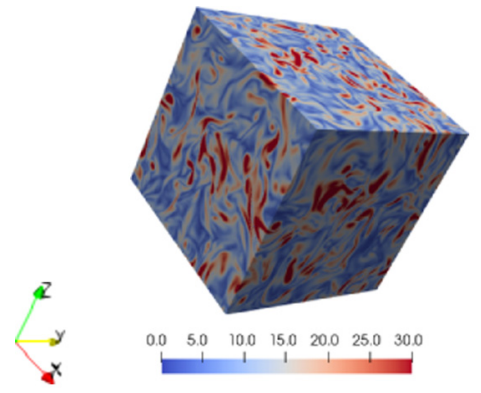

(a)

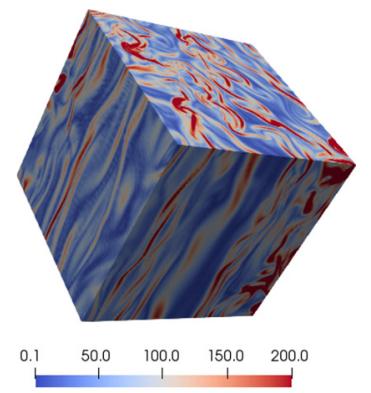

(b)

FIG. 1. Enstrophy in unstably stratified turbulence $(N=0.6$, $v=0.0067$ ). (a) Without magnetic field $[G=0$; see Eq. (11)]; (b) with magnetic field, $G=300$.

In order to reduce the importance of the nonlinear damping terms we add an additional source of anisotropy to the simulations, in the form of an imposed vertical magnetic field [F in Eq. (2)]. Indeed the Joule damping associated with the Lorentz force, rapidly tends to render the system invariant in the direction of the magnetic field if this latter is strong enough [41,42]. Equivalently, we could have added rotation to the system, as in [20], which also allows one to approach the 2D3C limit [43]. We consider the quasistatic approximation where the Lorentz force appears in closed form [42] in the Navier-Stokes equations, and the induction equation does not need to be solved. The Lorentz force acts then as a damping on the velocity fluctuations and its influence writes in this approximation, in Fourier representation,

$$
\hat{\boldsymbol{F}}=-\frac{B_{0}^{2}}{\eta \mu_{0}}\left(\cos ^{2} \phi\right) \hat{\boldsymbol{u}},
$$

with $\mu_{0}$ the permeability, $\eta$ the magnetic diffusivity, and $\phi$ the angle between the wave vector associated with $\hat{\boldsymbol{u}}$ and the direction of the magnetic field. This linear anisotropic damping term is of interest to gradually reach a 2D3C state. We fix the value $N=0.6$ and vary the value of $B_{0}$. The control

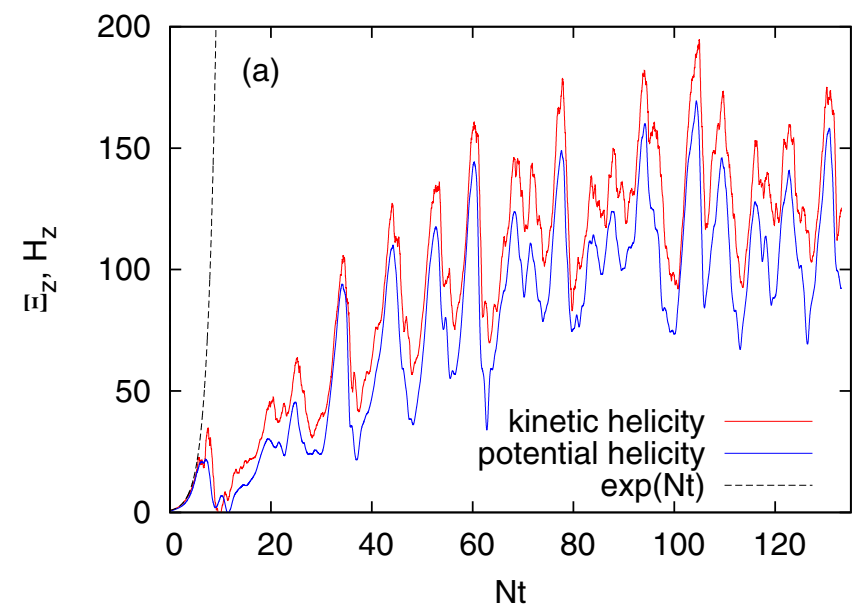

parameter is now the dimensionless number

$$
G=\frac{B_{0}^{2}}{N \eta \rho_{0} \mu_{0}},
$$

which is varied in the range $G \in[0,1100]$. For large values of $G$, the flow becomes almost invariant in the vertical direction [see Fig. 1(b)]. We quantify this by measuring the vertically averaged kinetic energy compared to the total kinetic energy as in [44]:

$$
\gamma=\frac{\langle E\rangle_{z}}{\langle E\rangle} \equiv \frac{\left\langle\left\|\frac{1}{L} \int_{0}^{L} \boldsymbol{u} d z\right\|^{2}\right\rangle}{\left\langle\|\boldsymbol{u}\|^{2}\right\rangle},
$$

which varies from $\gamma=0.6$ for $G=0$ to $\gamma=0.95$ for $G=$ 1100. This illustrates that the flow is close to the 2D3C limit for the largest values of $G$ we considered.

We show in Fig. 2(a), the results of a run where $G=300$, illustrating that there is helicity generation when the magnetic field is strong enough. Below $G \approx 40$ the value of the mean helicity could not easily be disentangled from random fluctuations.

The typical behavior for large values of $G$, as in Fig. 2(a), is a linear phase $(N t<5)$ for which the growth rate is exponential as predicted by the linearized equations (6)-(8), followed by a saturation when damping terms can no longer be neglected. Eventually helicity fluctuates around a steady state $(N t>100)$.

In the following we average helicity over the statistically steady state. Figure 2(b) shows the relative vertical helicity

$$
\tilde{H}_{z}=\left\langle u_{z} \omega_{z}\right\rangle /\left(\sqrt{\left\langle u_{z}^{2}\right\rangle} \sqrt{\left\langle\omega_{z}^{2}\right\rangle}\right) \text {. }
$$

This quantity does not exceed 0.1 , even for the largest values of the magnetic field strength. We will now give an explanation for this observation.

An intuitive, structure-based interpretation of helicity generation in the present flow is that the helicity corresponds to segregated patches of vertical vorticity with a definite sign of vorticity while they rise, and the opposite sign when they descend. The anisotropic damping by the magnetic field leads to an invariance in the vertical direction of these patches.

FIG. 2. (a) Time evolution of vertical kinetic helicity $H_{z}$ and potential helicity $\Xi_{z}$ for $N=0.6 ; G=300$. (b) Normalized helicities $\tilde{H}_{z}$, $\tilde{\Xi}_{z}$ as a function of $G$ (for fixed $N=0.6$ ).

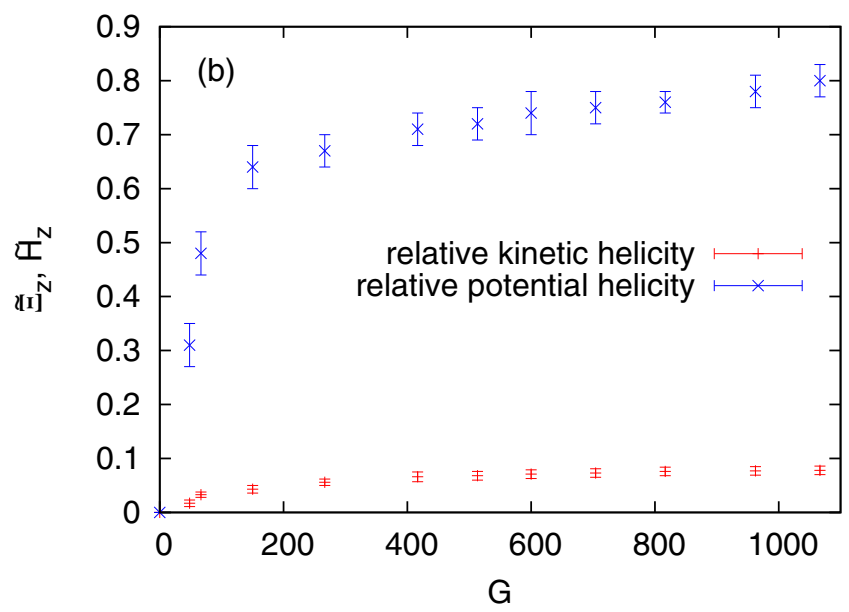




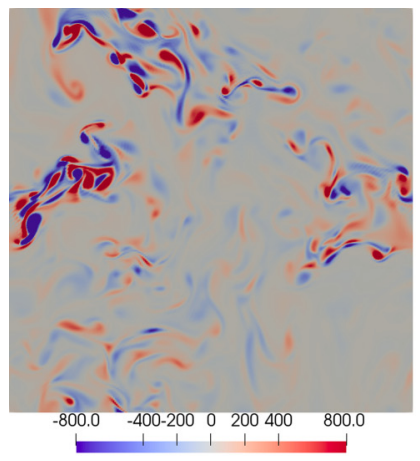

(a)

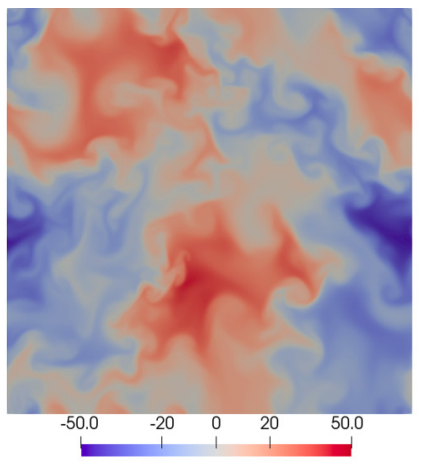

(b)

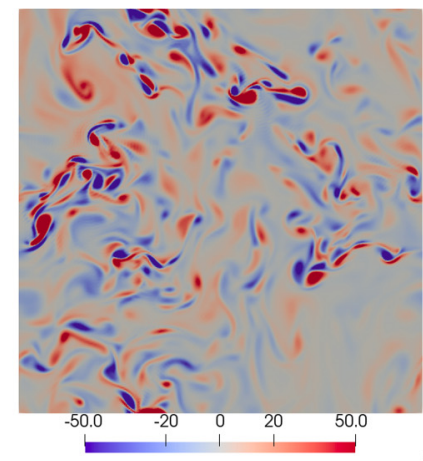

(c)

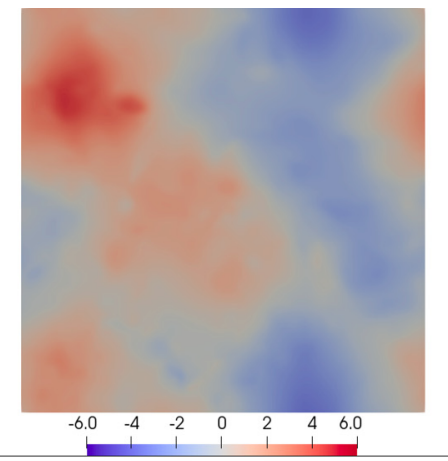

(d)

FIG. 3. Horizontal cuts through the simulation domain for $G=500$. The quantities are (a) vertical component of local helicity $h_{z}$, (b) vertical velocity $u_{z}$, (c) vertical vorticity $\omega_{z}$, and (d) stream function $\psi_{z}$ associated with the velocity in the horizontal plane.

This would correspond, in a horizontal cut through the domain, to a dominant value of the vertical helicity of one sign. This is assessed in Fig. 3(a). In this visualization, both positive and negative small-scale helicity patches $\left(h_{z}=u_{z} \omega_{z}\right)$ are observed. Furthermore, comparing the vertical velocity component Fig. 3(b) and vorticity Fig. 3(c), no clear spatial correlation is observed. It is thus not clear from these pictures which structures are responsible for the mean helicity. This visually confirms the small value of the relative helicity $\tilde{H}_{z}$.

It will be shown that the helicity is not contained in the small vortical structures visualized in Fig. 1(b) [or Fig. 3(c)], but in larger structures that are directly forced by the buoyancy instability driving the turbulence and which are associated with a two-dimensional condensate. With condensate we mean energy in the largest scales of a close to two-dimensional flow. In the Supplemental Material [40] it is shown that these large scales are close to a state where vorticity and velocity are perfectly aligned. Such a condensate results from blockage of the forward energy cascade for close-to-two-dimensional flow at large values of the magnetic field strength [45]. In order to highlight these large-scale structures in physical space, we introduce the stream function associated with the two-dimensional motion, $\psi_{z}$ such that $\omega_{z}=-\Delta_{x, y} \psi_{z}$, with $\Delta_{x, y}$ the horizontal Laplacian.

Indeed, both $\omega_{z}$ and $\psi_{z}$ are associated with rotating velocity patterns, but the vorticity is in general associated with smallscale structures, whereas the stream function characterizes larger-scale fluid motion. Visualizing $\psi_{z}$, in Fig. 3(d), it is observed that the large-scale rotating motion is fairly well correlated with the vertical velocity Fig. 3(b), which suggests that the mean helicity is contained in the large-scale, two-dimensional condensatelike structure of the flow. Indeed, considering the vorticity, it is almost impossible to discern the helicity containing structures of the flow.

To substantiate these observations, we introduce a new quantity named potential helicity (whereas the former helicity is designated by kinetic helicity) defined by

$$
\Xi_{z}=\left\langle u_{z} \psi_{z}\right\rangle
$$

This quantity is shown to evolve in time very similar to the kinetic helicity, as shown in Fig. 2(a).
The relative potential helicity

$$
\tilde{\Xi}_{z}=\left\langle u_{z} \psi_{z}\right\rangle /\left(\sqrt{u_{z}^{2}} \sqrt{\psi_{z}^{2}}\right)
$$

is, however, observed to be significantly larger than the kinetic helicity [Fig. 2(b)]. The fact that this quantity is close to unity and that the non-normalized value almost coincides with the kinetic helicity shows that the helicity is contained in the largest structures, associated with wave number $k \approx 1$, which is confirmed by the spectra shown in the Supplemental Material [40].

Note that in a different limit, namely, weak magnetohydrodynamic turbulence, for the imbalanced case with strong correlation between the magnetic field and the velocity field, condensates of the residual energy were observed, associated with the breakdown of mirror symmetry at the largest scales [46].

d. Reversals of the helicity. The presence of strong fluctuations could possibly allow for the reversal of the sign of the helicity. Indeed, both signs of the helicity are equally probable and it is natural to wonder whether the system can spontaneously reverse the sign of the helicity. We have investigated this possibility for small values of $G$, where the flow is not too close to its 2D3C limit. We show the results in Fig. 4 for $G=80$. We indeed observe reversals. We have shown in the visualizations and the spectra in the Supplemental Material [40] that the helicity is governed by the largest scales of the flow. The reversals seem therefore related to the stability of the flow structures associated with these scales. A complete statistical analysis of the reversals and the underlying dynamics is beyond the scope of this Letter.

e. Conclusions. We presented a generic mechanism to generate helicity without the need for walls, viscous effects, or statistical inhomogeneity. The generation of helicity is piloted by the interaction between skew diffusion and helicity, represented by Eqs. (6) and (8). For the mechanism to be effective, the flow needs the presence of an unstable density gradient and a body force which allows the system to attain a close-to-two-dimensional flow state.

The helicity is contained in condensatelike large-scale structures, associated with wave number $k=1$, which are better assessed using the stream function associated with the horizontal motion than using the vorticity. 


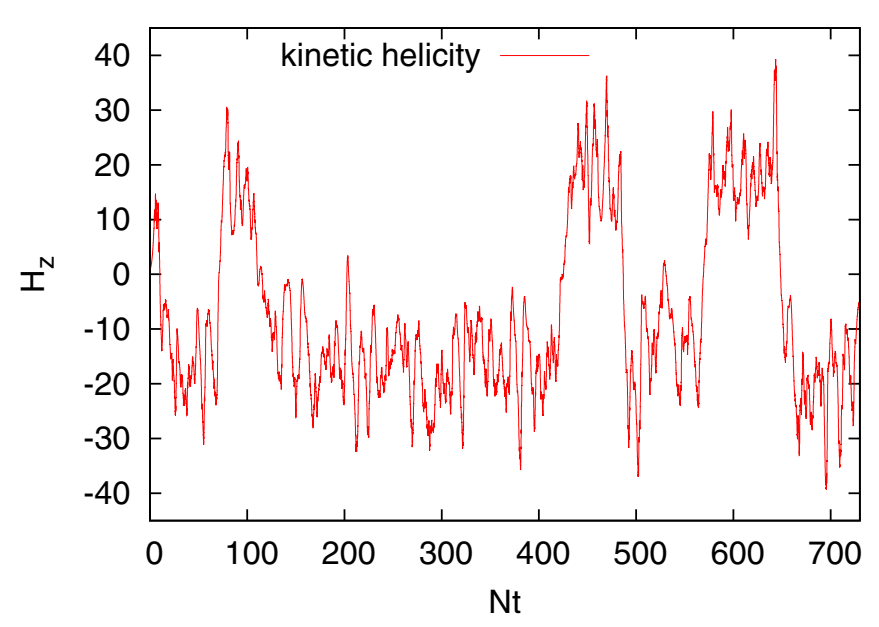

FIG. 4. Reversals of the value of the mean helicity can be observed for certain values of the magnetic field (here $G=80$ ).
The spontaneous nature of the helicity generation and the random reversals invite one to speculate on the relevance of the present observations for planetary dynamos and hurricane genesis. Considering the combination of unstable stratification with other effects such as system rotation seems a logical direction to further explore the present class of symmetry breaking and its possible geophysical relevance.

Acknowledgments. All simulations were carried out using the facilities of the PMCS2I (École Centrale de Lyon). We gratefully thank Laurent Pouilloux, Henri Lam, and Cenk Tekin for technical assistance. W.A. was partially supported by the French Federation for Magnetic Fusion Studies (FRFCM) and of the EUROfusion consortium from the Euratom research and training programme 2014-2018 and 2019-2020 under Grant Agreement No. 633053. The views and opinions expressed herein do not necessarily reflect those of the European Commission.
[1] J. J. Moreau, Constantes d'un îlot tourbillonnaire en fluide parfait barotrope, C. R. Hebd. Seances Acad. Sci. 252, 2810 (1961).

[2] H. K. Moffatt, The degree of knottedness of tangled vortex lines, J. Fluid Mech. 35, 117 (1969).

[3] H. K. Moffatt, Dynamo action associated with random inertial waves in a rotating conducting fluid, J. Fluid Mech. 44, 705 (1970).

[4] E. N. Parker, Cosmical Magnetic Fields: Their Origin and Their Activity (Oxford University Press, New York, 1979).

[5] B. Sreenivasan and C. A. Jones, Helicity generation and subcritical behaviour in rapidly rotating dynamos, J. Fluid Mech. 688, 5 (2011).

[6] D. K. Lilly, The structure, energetics and propagation of rotating convective storms. Part II: Helicity and storm stabilization, J. Atmos. Sci. 43, 126 (1986).

[7] J. Molinari and D. Vollaro, Rapid intensification of a sheared tropical storm, Mon. Weather Rev. 138, 3869 (2010).

[8] G. V. Levina and M. T. Montgomery, Numerical diagnosis of tropical cyclogenesis based on a hypothesis of helical selforganization of moist convective atmospheric turbulence, Dokl. Earth Sci. 458, 1143 (2014).

[9] A. Craya, Contribution à l'analyse de la turbulence associée à des vitesses moyennes, Ph.D. thesis, Université de Grenoble, 1957.

[10] J. R. Herring, Approach of axisymmetric turbulence to isotropy, Phys. Fluids 17, 859 (1974).

[11] C. Cambon and L. Jacquin, Spectral approach to non-isotropic turbulence subjected to rotation, J. Fluid Mech. 202, 295 (1989).

[12] F. Waleffe, The nature of triad interactions in homogeneous turbulence, Phys. Fluids A: Fluid Dyn. 4, 350 (1992).

[13] A. Alexakis, Helically decomposed turbulence, J. Fluid Mech. 812, 752 (2017).

[14] R. H. Kraichnan, Helical turbulence and absolute equilibrium, J. Fluid Mech. 59, 745 (1973).
[15] J. C. André and M. Lesieur, Influence of helicity on high Reynolds number isotropic turbulence, J. Fluid Mech. 81, 187 (1977).

[16] Q. Chen, S. Chen, and G. L. Eyink, The joint cascade of energy and helicity in three-dimensional turbulence, Phys. Fluids 15, 361 (2003).

[17] F. Plunian, A. Teimurazov, R. Stepanov, and M. K. Verma, Inverse cascade of energy in helical turbulence, J. Fluid Mech. 895, A13 (2020).

[18] A. Brissaud, U. Frisch, J. Léorat, M. Lesieur, and A. Mazure, Helicity cascades in fully developed isotropic turbulence, Phys. Fluids 16, 1366 (1973).

[19] P. D. Mininni and A. Pouquet, Helicity cascades in rotating turbulence, Phys. Rev. E 79, 026304 (2009).

[20] R. Marino, P. D. Mininni, D. Rosenberg, and A. Pouquet, Emergence of helicity in rotating stratified turbulence, Phys. Rev. E 87, 033016 (2013).

[21] K. Inagaki, N. Yokoi, and F. Hamba, Mechanism of mean flow generation in rotating turbulence through inhomogeneous helicity, Phys. Rev. Fluids 2, 114605 (2017).

[22] N. Yokoi and A. Brandenburg, Large-scale flow generation by inhomogeneous helicity, Phys. Rev. E 93, 033125 (2016).

[23] A. Gailitis, O. Lielausis, E. Platacis, S. Dement'ev, A. Cifersons, G. Gerbeth, T. Gundrum, F. Stefani, M. Christen, and G. Will, Magnetic Field Saturation in the Riga Dynamo Experiment, Phys. Rev. Lett. 86, 3024 (2001).

[24] R. Stieglitz and U. Müller, Experimental demonstration of a homogeneous two-scale dynamo, Phys. Fluids 13, 561 (2001).

[25] R. Monchaux, M. Berhanu, M. Bourgoin, M. Moulin, P. Odier, J.-F. Pinton, R. Volk, S. Fauve, N. Mordant, F. Pétrélis et al., Generation of a Magnetic Field by Dynamo Action in a Turbulent Flow of Liquid Sodium, Phys. Rev. Lett. 98, 044502 (2007).

[26] A. Pouquet and P. D. Mininni, The interplay between helicity and rotation in turbulence: Implications for scaling laws 
and small-scale dynamics, Philos. Trans. R. Soc. A 368, 1635 (2010).

[27] C. Rorai, D. Rosenberg, A. Pouquet, and P. D. Mininni, Helicity dynamics in stratified turbulence in the absence of forcing, Phys. Rev. E 87, 063007 (2013).

[28] V. Borue and S. A. Orszag, Spectra in helical three-dimensional homogeneous isotropic turbulence, Phys. Rev. E 55, 7005 (1997).

[29] F. G. Jacobitz, K. Schneider, W. J. T. Bos, and M. Farge, Influence of initial mean helicity on homogeneous turbulent shear flow, Phys. Rev. E 84, 056319 (2011).

[30] V. Dallas and S. M. Tobias, Forcing-dependent dynamics and emergence of helicity in rotating turbulence, J. Fluid Mech. 798, 682 (2016).

[31] P. H. Roberts and E. M. King, On the genesis of the Earth's magnetism, Rep. Prog. Phys. 76, 096801 (2013).

[32] N. Schaeffer, D. Jault, H.-C. Nataf, and A. Fournier, Turbulent geodynamo simulations: A leap towards Earth's core, Geophys. J. Int. 211, 1 (2017).

[33] P. A. Davidson and A. Ranjan, Are planetary dynamos driven by helical waves? J. Plasma Phys. 84, 735840304 (2018).

[34] P. A. Davidson and A. Ranjan, Planetary dynamos driven by helical waves-II, Geophys. J. Int. 202, 1646 (2015).

[35] H. K. Moffatt, Transport effects associated with turbulence with particular attention to the influence of helicity, Rep. Prog. Phys. 46, 621 (1983).

[36] A. Briard and T. Gomez, Dynamics of helicity in homogeneous skew-isotropic turbulence, J. Fluid Mech. 821, 539 (2017).
[37] In this $2 \mathrm{D} 3 \mathrm{C}$ limit, we note that the nontrivial relation between the contributions to the helicity $H_{z}=H_{x}+H_{y}$ can be derived using incompressibility.

[38] D. Lohse and F. Toschi, Ultimate State of Thermal Convection, Phys. Rev. Lett. 90, 034502 (2003).

[39] A. Burlot, B.-J. Gréa, F. S. Godeferd, C. Cambon, and J. Griffond, Spectral modelling of high Reynolds number unstably stratified homogeneous turbulence, J. Fluid Mech. 765, 17 (2015).

[40] See Supplemental Material at http://link.aps.org/supplemental/ 10.1103/PhysRevE.103.L061101 for description of the numerical code and parameters used, which includes [47].

[41] H. K. Moffatt, On the suppression of turbulence by a uniform magnetic field, J. Fluid Mech. 28, 571 (1967).

[42] B. Favier, F. S. Godeferd, C. Cambon, and A. Delache, On the two-dimensionalization of quasistatic magnetohydrodynamic turbulence, Phys. Fluids 22, 075104 (2010).

[43] B. Gallet, Exact two-dimensionalization of rapidly rotating large-Reynolds-number flows, J. Fluid Mech. 783, 412 (2015).

[44] B. Favier, L. J. Silvers, and M. R. E. Proctor, Inverse cascade and symmetry breaking in rapidly rotating Boussinesq convection, Phys. Fluids 26, 096605 (2014).

[45] S. Sundar, M. K. Verma, A. Alexakis, and A. G. Chatterjee, Dynamic anisotropy in MHD turbulence induced by mean magnetic field, Phys. Plasmas 24, 022304 (2017).

[46] S. Boldyrev and J. C. Perez, Spectrum of Weak Magnetohydrodynamic Turbulence, Phys. Rev. Lett. 103, 225001 (2009).

[47] T. Ishihara, K. Morishita, M. Yokokawa, A. Uno, and Y. Kaneda, Energy spectrum in high-resolution direct numerical simulations of turbulence, Phys. Rev. Fluids 1, 082403(R) (2016). 